OPEN ACCESS

Edited by: Ahmed Aliyu Abulfathi, Stellenbosch University, South Africa

Reviewed by: Evelyne Jacqz-Aigrain, Institut National de la Santé et de la Recherche Médicale (INSERM), France Kamunkhwala Gausi, University of Cape Town, South Africa

*Correspondence:

Muhammad Usman usman.ips@uvas.edu.pk

Specialty section: This article was submitted to Drugs Outcomes Research and

Policies,

a section of the journal Frontiers in Pharmacology

Received: 07 June 2021 Accepted: 12 October 2021 Published: 11 November 2021

Citation: Munir MM, Rasheed H, Khokhar MI, Khan RR, Saeed HA, Abbas M, Ali M, Bilal R, Nawaz HA, Khan AM, Qamar S, Anjum SM and Usman M (2021) Dose

Tailoring of Vancomycin Through Population Pharmacokinetic Modeling Among Surgical Patients in Pakistan.

Front. Pharmacol. 12:721819.

doi: 10.3389/fphar.2021.721819

\section{Dose Tailoring of Vancomycin Through Population Pharmacokinetic Modeling Among Surgical Patients in Pakistan}

\author{
Muhammad Muaaz Munir ${ }^{1}$, Huma Rasheed ${ }^{1}$, Muhammad Imran Khokhar ${ }^{2}$, \\ Rizwan Rasul Khan ${ }^{3}$, Hafiz Asad Saeed ${ }^{4}$, Mateen Abbas ${ }^{5},{\text { Mohsin } \text { Ali }^{6}, \text { Rabiea Bilal }}^{7}$, \\ Hafiz Awais Nawaz ${ }^{1}$, Abdul Muqeet Khan ${ }^{5}$, Shaista Qamar ${ }^{1}$, Syed Muneeb Anjum ${ }^{1}$ and \\ Muhammad Usman ${ }^{1 *}$ \\ ${ }^{1}$ Institute of Pharmaceutical Sciences, University of Veterinary and Animal Sciences, Lahore, Pakistan, ${ }^{2}$ Ameer-ud-Din Medical \\ College, Post-Graduate Medical Institute (PGMI), Lahore General Hospital, Lahore, Pakistan, ${ }^{3}$ Department of Medicine, Aziz \\ Fatima Medical and Dental College, Faisalabad, Pakistan, ${ }^{4}$ Pak Emirates Military Hospital, Rawalpindi, Pakistan, ${ }^{5}$ Quality \\ Operation Laboratory, University of Veterinary and Animal Sciences, Lahore, Pakistan, ${ }^{6}$ Department of Pharmacy Practice, \\ Faculty of Pharmaceutical Sciences, Govt College University, Faisalabad, Pakistan, ${ }^{7} \mathrm{CMH}$ Lahore Medical College and IOD, \\ NUMS, Lahore, Pakistan
}

Background: Vancomycin is a narrow therapeutic agent, and it is necessary to optimize the dose to achieve safe therapeutic outcomes. The purpose of this study was to identify the significant covariates for vancomycin clearance and to optimize the dose among surgical patients in Pakistan.

Methods: Plasma concentration data of 176 samples collected from 58 surgical patients treated with vancomycin were used in this study. A population pharmacokinetic model was developed on $\mathrm{NONMEM}^{\circledR}$ using plasma concentration-time data. The effect of all available covariates was evaluated on the pharmacokinetic parameters of vancomycin by stepwise covariate modeling. The final model was evaluated using bootstrap, goodness-of-fit plots, and visual predictive checks.

Results: The pharmacokinetics of vancomycin followed a one-compartment model with first-order elimination. The vancomycin clearance (CL) and volume of distribution (Vd) were $2.45 \mathrm{~L} / \mathrm{h}$ and $22.6 \mathrm{I}$, respectively. Vancomycin $\mathrm{CL}$ was influenced by creatinine clearance (CRCL) and body weight of the patients; however, no covariate was significant for its effect on the volume of distribution. Dose tailoring was performed by simulating dosage regimens at a steady state based on the CRCL of the patients. The tailored doses were 400, 600, 800 , and $1,000 \mathrm{mg}$ for patients with a CRCL of $20,60,100$, and $140 \mathrm{ml} / \mathrm{min}$, respectively.

Conclusion: Vancomycin CL is influenced by CRCL and body weight of the patient. This model can be helpful for the dose tailoring of vancomycin based on renal status in Pakistani patients.

Keywords: vancomycin, population pharmacokinetics, Pakistan, NONMEM, dose tailoring 


\section{INTRODUCTION}

Vancomycin has been used for more than six decades and is predominantly effective for the treatment of infections caused by methicillin-resistant Staphylococcus aureus (MRSA), methicillinsusceptible Staphylococcus aureus (MSSA), and Staphylococcus epidermidis (Zhao et al., 2014). Plasma levels of vancomycin below the target trough concentration may cause insufficient eradication of bacteria, while overdosing may lead to toxicity (Ingram et al., 2008). The therapeutic trough concentration of vancomycin is a crucial pharmacokinetic metric to achieve the antibacterial effect and reduction in microbial resistance. Therefore, therapeutic drug monitoring (TDM) is recommended for the safe and effective outcomes of vancomycin treatment (Martin et al., 2010). The recently published consensus guidelines by the American Society of Health-System Pharmacists (ASHP), Infectious Diseases Society of America (IDSA), the Pediatric Infectious disease Society (PIDS), and the Society of Infectious Diseases Pharmacists (SIDP) have suggested vancomycin trough concentrations of $15-20 \mathrm{mg} / \mathrm{l}$ for most infections (Rybak et al., 2020).

Age-related differences in the pharmacokinetics of vancomycin in pediatric and adult patients necessitate dose adjustment. Developmental changes in kidney function also affect the serum concentrations of drugs that are renally excreted, as in the case of vancomycin, where $80 \%-90 \%$ of the drug is excreted via renal elimination (Matzke et al., 1984; Rodvold et al., 1988; Ducharme et al., 1994). Therefore, the kidney status of patients needs special attention for proper vancomycin dosing, particularly in elderly patients exhibiting a progressive decline in kidney function with increasing age (Verhave et al., 2005). The between-subject variability necessitates dose individualization by considering patientspecific characteristics such as body weight, age, and ongoing organ maturation, which makes population pharmacokinetics a valuable approach.

Population pharmacokinetic analyses of vancomycin have been performed in a variety of disease conditions, including patients with sepsis or septic shock (Heffernan et al., 2019), critically ill patients (Llopis-Salvia and Jiménez-Torres, 2006; Udy et al., 2013; Escobar et al., 2014; Petejova et al., 2014), severely burnt patients (Yang et al., 2007; Dolton et al., 2010), cancer patients (Buelga et al., 2005; Omote et al., 2009; Al-Kofide et al., 2010), extremely obese patients (Grace, 2012; Adane et al., 2015), patients with various degrees of renal function (Rodvold et al., 1988; Staatz et al., 2006; Kim et al., 2019; Chu et al., 2020), patients with peritonitis (Montanes Pauls et al., 2011), and during hydrocephalic shunt prophylaxis (Leroux et al., 1990). Population pharmacokinetics have also been widely studied in different age groups, including geriatric patients (Usman et al., 2018; Zhou et al., 2019) and pediatric patients under various clinical conditions (Anderson et al., 2007; Machado et al., 2007; Lo et al., 2010; Badran et al., 2011; Giachetto et al., 2011; Stockmann et al., 2013a; Mahmoud et al., 2014; Zhao et al., 2014; Moffett et al., 2019a; Moffett et al., 2019b; Moffett et al., 2019c). Moreover, studies in different ethnic groups are also available regarding the population pharmacokinetics of vancomycin (Purwonugroho et al., 2012; Deng et al., 2013; Zhou et al., 2019). Simulations of different dosage regimens to maintain trough concentrations of vancomycin between 10 and $20 \mathrm{mg} / \mathrm{l}$ have been performed in different clinical cases such as critically ill patients (Kovacevic et al., 2020), pneumonia patients (Yamamoto et al., 2009), and patients with hematological malignancies (Buelga et al., 2005). The covariates considered for dosing simulations in different studies have included highvolume hemofiltration (Escobar et al., 2014) serum cystatin C (Chung et al., 2013), and creatinine clearance (Purwonugroho et al., 2012). However, to the best of our knowledge, based on a literature search, no population pharmacokinetic study of vancomycin has been reported in Pakistani patients, and this is probably the first study on the population pharmacokinetics of vancomycin in Pakistani patients using a non-linear mixed effect (NONMEM) modeling approach. Pakistan, with more than 200 million people (Pakistan Bureau of Statistics, 2017), exhibits diversity in its population with regard to lifestyle, eating habits, genetic polymorphism, etc. Since no population pharmacokinetic study of vancomycin in Pakistani patients has been reported so far, this study aimed to develop a population pharmacokinetic model of vancomycin in adult Pakistani patients by using NONMEM $^{\circledR}$ and to evaluate the influence of different covariates on pharmacokinetic parameters. Furthermore, the simulations of dosage regimens were performed using the population pharmacokinetic model, which was developed using data obtained from Pakistani patients rather than from the literature.

\section{MATERIALS AND METHODS}

\section{Patient selection and sampling}

A total of 176 blood samples were collected from 58 patients admitted to the surgical unit of Lahore General Hospital, Pakistan, between August and December 2018. The study was approved by the Institutional Review Committee (IRC) for Biomedical Research of the University of Veterinary and Animal Sciences, Lahore, Pakistan, vide notification no. 018/ IRC/BMR. The sample collection was conducted in accordance with the Declaration of Helsinki for medical research involving human subjects (World Medical Association, 2001). Only patients who underwent a major surgical procedure and were above the age of 18 years were included in the study. Written informed consent was obtained from all patients or their close relatives. Vancomycin was administered via IV infusion for $0.5 \mathrm{~h}$. The dose selection and duration of infusion were at the discretion of the attending medical practitioners. Blood samples were collected at different time intervals after administration of the first dose of vancomycin, and the samples were centrifuged at $5,000 \mathrm{rpm}$ for $5 \mathrm{~min}$ to separate the plasma, which was stored at $-20^{\circ} \mathrm{C}$ until analysis. Patient demographics, including age, weight, sex, type of disease, and serum creatinine (SCR) were recorded from patient files, and creatinine clearance (CRCL) was calculated using the Cockcroft and Gault equation 
(Cockcroft and Gault, 1976). The patients demographics and sampling record is provided in Table $\mathbf{1 .}$

\section{Sample analysis}

The concentrations of vancomycin in individual samples were quantified at the Quality Operation Laboratory of the University of Veterinary and Animal Sciences, Lahore, using an already developed and validated HPLC method (Usman and Hempel, 2016). Briefly, separation was carried out using a C18 column $(125 \mathrm{~mm} \times 4.6 \mathrm{~mm}$, $5 \mu \mathrm{m})$ with UV detection at $205 \mathrm{~nm}$. The mobile phase was a combination of buffer (50 $\mathrm{mM}$ ammonium dihydrogen phosphate) at $\mathrm{pH} 2.2$ and acetonitrile $(88: 12 \mathrm{v} / \mathrm{v})$. The method was linear over the concentration range of $0.25-60 \mathrm{mg} / \mathrm{l}$. The lower limit of quantification was $0.25 \mathrm{mg} / \mathrm{l}$ with relative standard deviations (RSD) of $17.8 \%$ and $3.03 \%$ for inter-day and intra-day precision, respectively.

\section{Population pharmacokinetic modeling}

The population pharmacokinetic model was developed using nonlinear mixed effect modeling (NONMEM version 7.4.4) software provided by Icon Clinical Research LLC, New York, NY, USA, along with the Perl-speaks-NONMEM (PsN) toolkit (Lindbom et al., 2005). The execution and management of the model as well as report generation were performed with the aid of Pirana (Certara, Princeton, NJ, USA) (Keizer et al., 2011). The actual process of modeling began with the development of onecompartment and two-compartment base models using the first-order conditional estimation method with interaction (FOCE-I) to obtain pharmacokinetic parameter estimates without any covariates. The allometric scaling function was used to evaluate the relationship between body weight and vancomycin clearance $(\mathrm{CL})$ as well as volume of distribution $(\mathrm{Vd})$, with a coefficient of 0.75 for $\mathrm{CL}$ and 1 for $\mathrm{Vd}$. The exponential random-effect model was used to describe the between-subject variability (BSV) on pharmacokinetic (PK) parameters, while additive, proportional, and combined residual error models were tested to describe the residual error between the observed and predicted concentrations of vancomycin.

\section{Covariate analysis}

The influence of different covariates (age, sex, WT, SeCR, and CRCL) on CL and Vd was determined by stepwise covariate modeling (SCM) in which the covariates were included in the model by forward inclusion and backward elimination processes. During the forward inclusion step, all the tested covariates were added to the base model in a stepwise manner, and the influence on the objective function value (OFV) of the model was observed. For a tested covariate, a drop of 3.84 points in OFV $(\alpha=0.05)$ between the two nested models was considered significant for inclusion. The process was repeated until no significant covariate was available for inclusion in the full model. During the backward elimination step, the added covariates in the full model were eliminated one by one, and an increase in OFV was observed with a stricter criterion for level of significance $(\alpha=0.01)$. A rise in OFV $\geq 6.63$ points was considered significant for the retention of covariates in the final model.

\section{Model evaluation}

A visual observation of the goodness-of-fit plots was performed to evaluate the predictive performance of the final model. The
TABLE 1 | Patients and sample data.

\begin{tabular}{lc}
\hline Patient demographic data & Median (range) \\
\hline No. of patients & 58 \\
Male (\%) & $39(73.7)$ \\
Female (\%) & $19(26.3)$ \\
Age (years) & $54(25-86)$ \\
Weight (kg) & $75(53-129)$ \\
SeCr (mg/dL) & $0.935(0.4-4.7)$ \\
CRCL (mL/min) & $101.15(15.9-177.2)$ \\
Types of patients & - \\
Patients with grossly contaminated wound & $13(34.2 \%)$ \\
Gas gangrene patients & $18(47.4 \%)$ \\
Patients with severe peritonitis & $7(18.4 \%)$ \\
Sample data & - \\
No. of samples & 176 \\
Samples per patient (average) & $3(1-7)$ \\
Dose (mg) & $500-1,000$ \\
Concentration (mg/L) & $23.5(1.9-52.6)$
\end{tabular}

goodness-of-fit plots included the scatterplots of observed concentrations (DV) versus population-predicted concentrations (PRED), DV versus individual predicted concentrations (IPRED), conditional weighted residuals (CWRES) versus PRED, and CWRES versus time after dose. The robustness and stability of the final model were evaluated by bootstrap analysis with 1,000 datasets generated by repeated sampling from the final model with different combinations of subjects. The median values of bootstrap estimates along with $95 \%$ confidence intervals (based on the 2.5th and 97.5th percentiles) were compared with the respective parameter estimates of the final model.

A visual predictive check (VPC) was performed to evaluate the predictive performance of the final model. The observed concentrations along with the median and 5th and 95th percentiles were overlaid on the $90 \%$ prediction interval (5th and 95th percentiles) of simulated concentrations obtained from 500 virtual datasets of the final model.

\section{Dosing simulations}

As vancomycin is primarily eliminated via the kidney through glomerular filtration, dosing simulations were performed based on the renal status of the patients. For this purpose, four virtual patients were simulated with CRCL levels of $20,60,100$, and $140 \mathrm{ml} / \mathrm{min}$. Initially, the same dose [1,000 mg every $12 \mathrm{~h}$ (q12h)] was used to simulate 1,000 plasma concentrations at a steady state for all four virtual patients by keeping the target trough concentration range (TTCR) between 10 and $20 \mathrm{mg} / \mathrm{l}$. After visual observation of simulated concentrations, the tailored dose was used, and plasma concentrations were simulated for 1,000 patients at each level of CRCL.

\section{RESULTS}

\section{Patient demographics and exploratory data analysis}

The plasma concentration data for 58 patients ( 39 male and 19 female) with 176 samples were used for the development of the base model. The median age and body weight of the patients were 
54 years and $75 \mathrm{~kg}$, ranging from 25 to 86 years and $53-129 \mathrm{~kg}$, respectively. The median serum creatinine level was $0.935 \mathrm{mg} / \mathrm{dl}$ with a range of $0.4-4.7 \mathrm{mg} / \mathrm{dl}$, while the median creatinine clearance calculated by the Cockcroft and Gault equation was $101.15 \mathrm{ml} / \mathrm{min}$, ranging from 15.9 to $177.2 \mathrm{ml} / \mathrm{min}$. The majority of patients received a 1,000-mg dose of vancomycin administered over a $0.5 \mathrm{~h}$ infusion.

\section{Population PK modeling}

One-and two-compartment models were used for the data analysis. However, the two-compartment model was not able to predict inter-compartmental clearance $(\mathrm{Q})$ or volume of the peripheral compartment $\left(\mathrm{V}_{2}\right)$ in a stable manner. Moreover, the values of the fixed-effect and random-effect parameters were also not stable in the two-compartment models (results not shown). Therefore, the one-compartment model with first-order elimination (ADVAN1 TRANS2) was used for the development of the base model. The initial value of OFV for the base model was 729.9 while the OFV of the final model after the inclusion of significant covariates was 670.1 , which is 59.8 points less than the base model. The BSV for vancomycin CL and $\mathrm{Vd}$ was described by an exponential random effect, while residual variability between the observed and predicted vancomycin concentrations was described by a proportional error model. The BSV for vancomycin CL and Vd were $11.3 \%$ and $22.8 \%$, respectively, while the additive error was 3.07 .

\section{Covariate analysis}

Stepwise covariate modeling showed that CRCL and patient body weight were significant covariates for vancomycin CL and reduced OFV by 42.01 and 9.13 points, respectively, after inclusion in the base model. In the final model, the values for vancomycin $\mathrm{CL}$ and $\mathrm{Vd}$ were $2.45 \mathrm{~L} / \mathrm{h}$ and $22.6 \mathrm{~L}$, respectively. The BSV for vancomycin CL decreased from $26.6 \%$ to $9.14 \%$ after the inclusion of covariates. Eq. 1 describes the influence of CRCL and body weight on vancomycin CL in the final model.

$$
\mathrm{CL}_{\mathrm{j}}=2.45 \times \mathrm{CLCRCL} \times \mathrm{CLWT} \times \mathrm{e}^{\eta 1}
$$

where $\mathrm{CL}_{\mathrm{j}}$ is the vancomycin clearance for the $j$ th individual, $\eta_{1}$ is the BSV for vancomycin CL, and 2.45 is the median value of vancomycin CL for this population.

$$
\begin{gathered}
\mathrm{CLCRCL}=\left(1+0.0046 \times\left(\mathrm{CRCL}_{\mathrm{j}}-101.15\right)\right) \\
\mathrm{CLWT}=\left(1-0.011 \times\left(\mathrm{WT}_{\mathrm{j}}-75\right)\right)
\end{gathered}
$$

where $\mathrm{CRCL}_{\mathrm{j}}$ and $\mathrm{WT}_{\mathrm{j}}$ are the CRCL and weight of the $j$ th individual while 101.15 and 75 are the median values of CRCL and weight in the population, respectively.

The inter-relationships between CL versus age and CL versus CRCL are shown in Figure 1. The CL of vancomycin decreased continuously with age (Figure 1A), while it increased in direct relation with CRCL (Figure 1B).

Although the age of the patient was not a significant covariate for vancomycin CL, a categorical decline in vancomycin CL with increasing age was observed when age was stratified into three different groups: $\leq 45$ years, 46-60 years, and '60 years
(Figure 2A). A categorical increase in vancomycin CL was also observed with increasing CRCL when the patients were divided into three different groups based on CRCL: $\leq 60 \mathrm{ml} /$ $\mathrm{min}, 60-120 \mathrm{ml} / \mathrm{min}$, and ${ }^{>} 120 \mathrm{ml} / \mathrm{min}$ (Figure 2B).

\section{Model evaluation}

The combined goodness-of-fit plots of DV versus PRED, DV versus IPRED, CWRES versus PRED, and CWRES versus time after dose are shown in Figures 3A-D. A uniform distribution of observed concentrations (DV) and predicted concentrations (PRED and IPRED) was observed around the line of identity (Figures 3A and B). Moreover, a random distribution of CWRES values around the zero line was observed, and 95\% of the CWRES values were within the range of -2 to 2 in the final model (Figures 3C and D).

The parameter estimates of the final model compared with the median values of 1,000 bootstrap estimates along with $95 \%$ confidence intervals based on the 2.5th and 97.5th percentiles are given in Table 2. The values for bootstrap estimates were close to the parameter estimates of the final model, with a bias value of $\leq 7.25 \%$.

The VPC revealed that the observed concentrations of vancomycin along with median and 5th and 95th percentiles were within the $90 \%$ prediction interval of the simulated concentrations, as shown in Figure 4.

\section{Dosing simulations}

The results of the vancomycin dosing simulation at four levels of CRCL are shown in Figure 5. Administration of a similar dose (1,000 $\mathrm{mg} \mathrm{q12h)}$ to all patients resulted in average vancomycin trough concentrations of $33.3,23.9,18.0$, and $13.7 \mathrm{mg} / \mathrm{l}$ for patients with CRCL 20,60,100, and $140 \mathrm{ml} / \mathrm{min}$, respectively (Table 3).

The vancomycin concentrations for patients with CRCL levels of 20 and $60 \mathrm{ml} / \mathrm{min}$ were above the TTCR, while patients with a CRCL of $100 \mathrm{ml} / \mathrm{min}$ maintained trough concentrations near the upper limit of TTCR (Figure 5A). After attempting multiple dosing regimens, 400,600, 800, and 1,000 mg were found to be suitable for patients with CRCL of $20,60,100$, and $140 \mathrm{ml} / \mathrm{min}$, respectively, to maintain the trough concentration of vancomycin between 10 and $20 \mathrm{mg} / \mathrm{l}$ (Figure 5B).

\section{DISCUSSION}

Vancomycin is a narrow therapeutic index drug, and dose consideration is highly important for safe and effective treatment outcomes. The identification of patient characteristics and the magnitude of variability among individuals that influence the pharmacokinetics of a drug in a particular population can be helpful for dose individualization. As $80 \%-90 \%$ of administered vancomycin is eliminated through the kidney (Matzke et al., 1984; Rodvold et al., 1988; Ducharme et al., 1994), patients with augmented renal function (as in the case of increased cardiac output in the early stage of severe sepsis) are at high risk of sub-therapeutic plasma concentrations of vancomycin, which may result in therapeutic failure. On the 

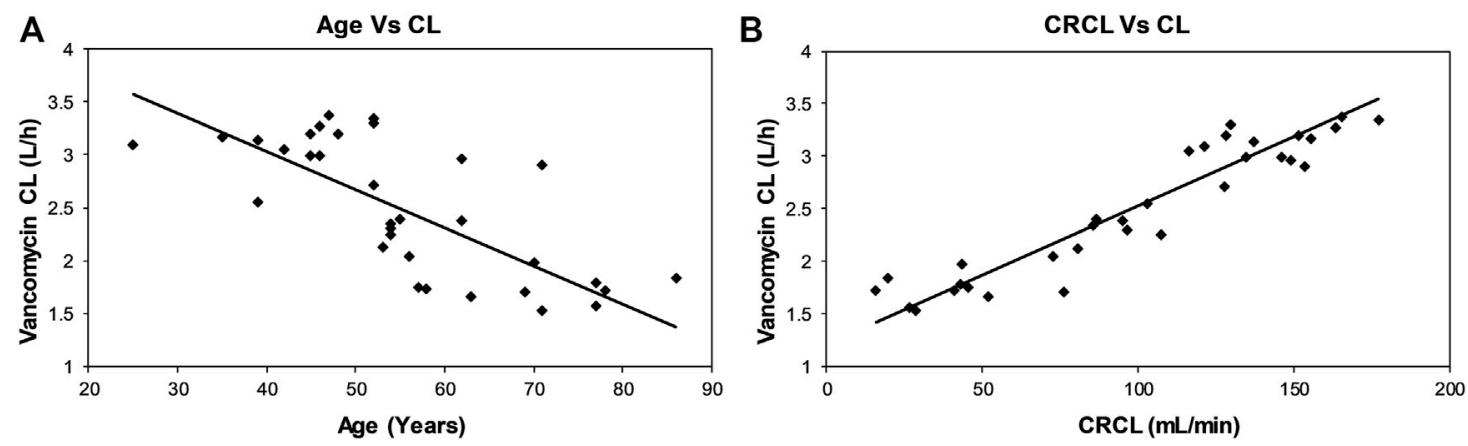

FIGURE 1 | Scatterplots showing relationships between vancomycin CL and age (A) and CRCL (B) of the patients.
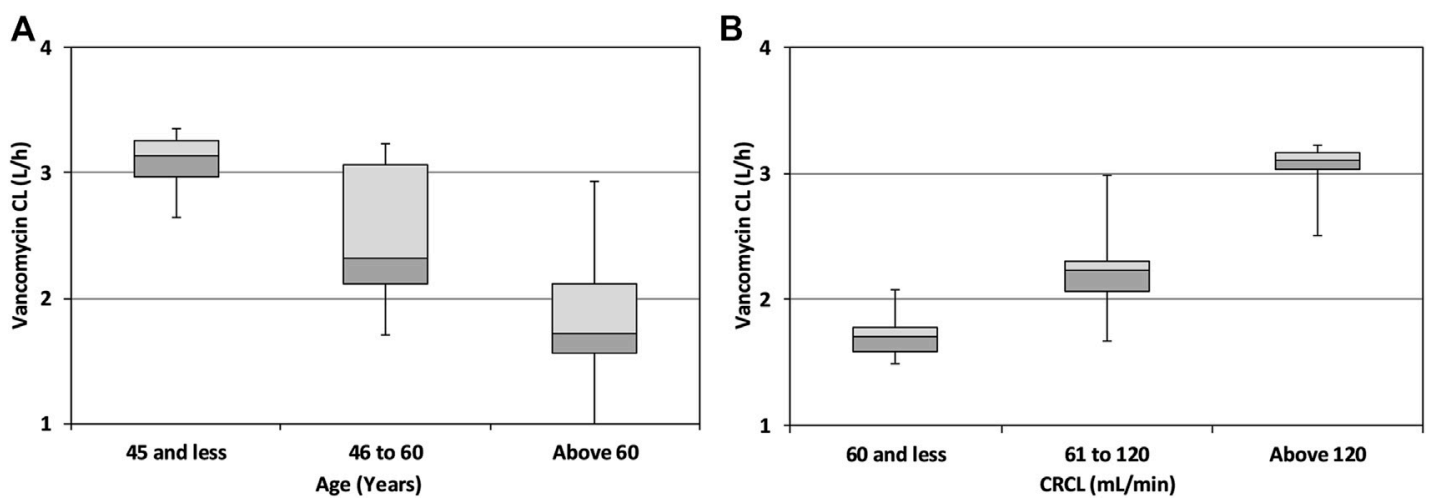

FIGURE 2 | Box and whisker plots showing categorical change in vancomycin CL with age (A) and CRCL (B) of the patients after stratification into three groups.

other hand, patients with decreased renal function are at a risk of drug accumulation and toxicity (Taccone et al., 2011; Varghese et al., 2011). The selection of a dosage regimen is important for the safe and effective use of drugs in a target patient population or, more specifically, in an individual patient (Owen and FiedlerKelly, 2014). Population pharmacokinetic modeling is the study of variability among individuals with respect to drug concentrations after the administration of clinically relevant dosage regimens (Aarons, 1991), and it can be used to construct dosing strategies for individual patients (Ette and Williams, 2004).

Many studies have been conducted on the population pharmacokinetics of vancomycin in different countries and under different conditions. The majority of the studies were carried out in high-income countries, and few were conducted in upper-middle-income countries (UMICs) (Lo et al., 2010; Purwonugroho et al., 2012; Lin et al., 2016; Chen et al., 2018; Li et al., 2018), while only one study was available in a lowermiddle-income country (Abdel Hadi et al., 2016). To the best of our knowledge, this is the first study to investigate the population pharmacokinetics of vancomycin in Pakistani patients.

The description of data by one compartment with first-order elimination is in line with the majority of reported population pharmacokinetic studies (Kimura et al., 2004; Buelga et al., 2005; Anderson et al., 2007; Lo et al., 2010; Tanaka et al., 2010;
Stockmann et al., 2013a; Udy et al., 2013; Zhao et al., 2014; Adane et al., 2015; Usman et al., 2018; Moffett et al., 2019c; Heffernan et al., 2019; Zhou et al., 2019). However, twocompartment models were used to determine the population pharmacokinetics of vancomycin in some studies (Grimsley and Thomson, 1999; Thomson et al., 2009; Beumier et al., 2013; Escobar et al., 2014). The mean value of vancomycin CL in Pakistani patients was $2.45 \mathrm{~L} / \mathrm{h}$, which is in close agreement with the reported CL values of $1.08-2.99 \mathrm{~L} / \mathrm{h}$ in other studies (Buelga et al., 2005; Llopis-Salvia and Jiménez-Torres, 2006; Staatz et al., 2006; Thomson et al., 2009; Tanaka et al., 2010; Udy et al., 2013; Escobar et al., 2014; Usman et al., 2018). However, obese patients observed in different studies exhibited high values of vancomycin CL, from 6.54 to $11.8 \mathrm{~L} / \mathrm{h}$ (Blouin et al., 1982; Bauer et al., 1998; Adane et al., 2015). The BSV for vancomycin CL in Pakistani patients was $11.3 \%$, which is less than the reported range from $16 \%$ (Staatz et al., 2006) to $40.6 \%$ (Seay et al., 1994). However, a lower value of BSV for CL of $4.5 \%$ has been reported in a study conducted on 1812 patients with end-stage renal disease (ESRD) (Goti et al., 2018). In the covariate analysis, CRCL and patient weight were significant covariates for vancomycin CL. As the major route of vancomycin elimination is the kidney, changes in CRCL can influence vancomycin CL. Body weight is another covariate that significantly affected vancomycin CL in Pakistani patients, possibly 

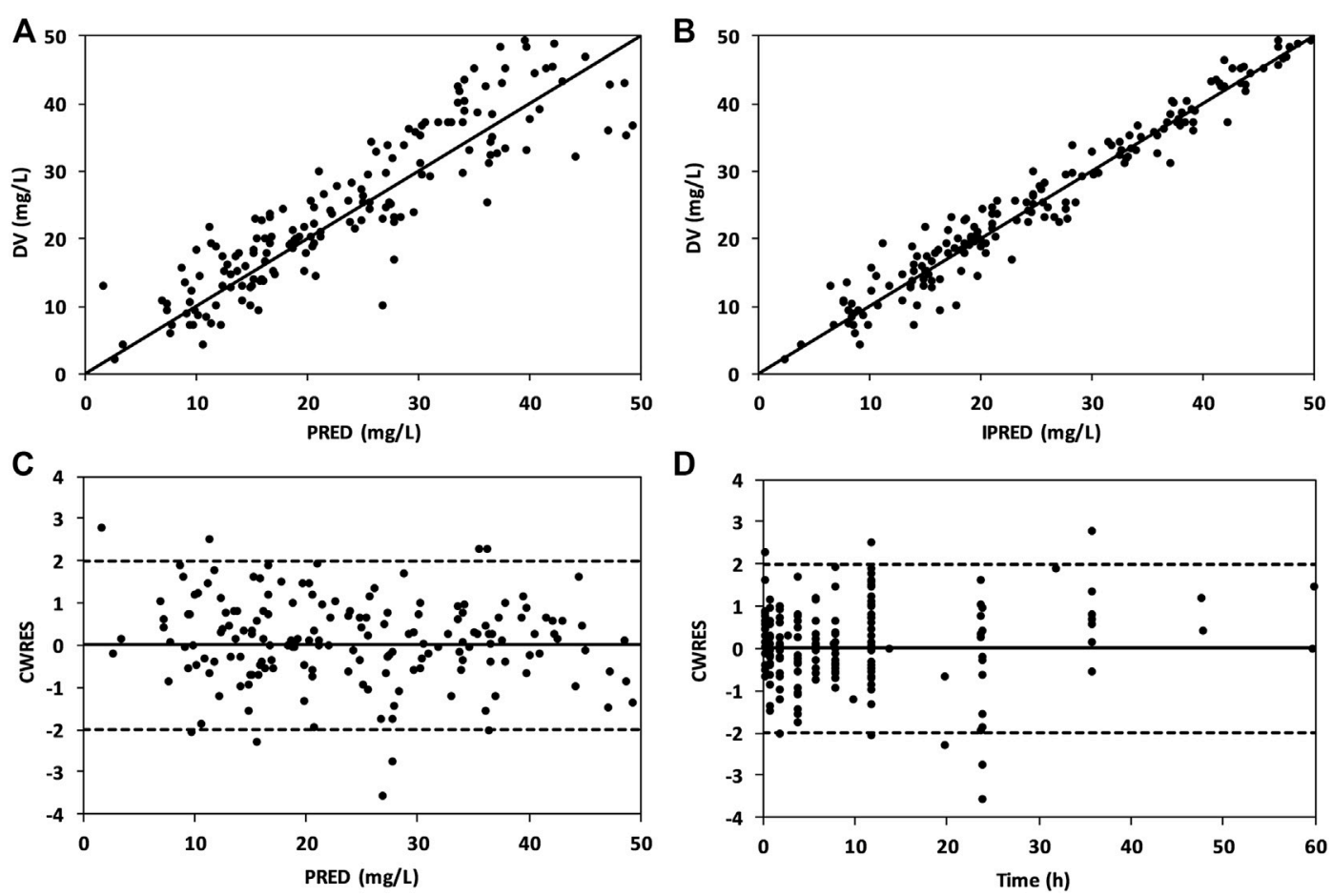

FIGURE 3 | Goodness-of-fit plots of the final model showing DV vs. PRED (A), DV vs. IPRED (B), CWRES vs. PRED (C), and CWRES vs. time after dose (D).

TABLE 2 | Population parameter estimates compared with 1,000 bootstrap estimates

\begin{tabular}{|c|c|c|c|c|c|}
\hline Parameter & Final estimate & RSE \% & Bootstrap estimate & $95 \% \mathrm{Cl}^{\mathrm{a}}$ & Bias \% \\
\hline$C L(L / h)^{b}$ & 2.45 & 2 & 2.46 & $2.35-2.59$ & -0.21 \\
\hline$V(\mathrm{~L})$ & 22.6 & 5 & 22.7 & 20.3-26.1 & -0.39 \\
\hline$C L-W T^{d}$ & 0.011 & 10 & 0.0107 & $0.0087-0.013$ & 2.15 \\
\hline BSV-CL $(\%)^{e}$ & 11.3 & 38 & 10.48 & $4.18-14.4$ & 7.25 \\
\hline $\mathrm{BSV}-\mathrm{Vd}^{\mathrm{e}}(\%)$ & 22.8 & 51 & 21.8 & $13.7-53.1$ & 4.38 \\
\hline
\end{tabular}

${ }^{\text {a } 95 \% ~} \mathrm{Cl}$ based on the 2.5 th to 97.5 th percentiles of distribution.

${ }^{b}$ Clearance at a CRCL of $101.15(\mathrm{~mL} / \mathrm{min})$ and weight of $75 \mathrm{~kg}$.

${ }^{c}$ Proportional change in $C L$ with $C R C L$.

${ }^{d}$ Proportional change in $C L$ with weight of the patients.

${ }^{e} B$ Between subject variability of $C L$ and $V d$ expressed in percentage coefficient of variation.

due to the diversity in body weight of patients used in this study (53-129 kg with a median of $75 \mathrm{~kg}$ ). A few studies have also reported the influence of age on CL (Revilla et al., 2010; Chung et al., 2013). In neonates and children, body weight (Kimura et al., 2004; Stockmann et al., 2013b; Zhao et al., 2014; Guilhaumou et al., 2016; Li et al., 2018), gestational age (Grimsley and Thomson, 1999; Kimura et al., 2004; Lo et al., 2010), and postnatal age (PNA) (Anderson et al., 2007; Zhao et al., 2014; Stockmann et al., 2015; Chen et al., 2018) have been reported as significant covariates for vancomycin CL. The Vd for vancomycin was 22.61 (BSV 16.1\%), as compared with a reported range in Vd from 11.81 (Escobar et al., 2014) to 1011 (Lin et al., 2016). However, no covariate was shown to be significant for $\mathrm{Vd}$ of vancomycin in our population.
The simulations of vancomycin dosing based on the renal status of patients indicated that $800-1,000 \mathrm{mg}$ of vancomycin administered every $12 \mathrm{~h}$ is sufficient for maintaining the trough concentration of vancomycin between 10 and $20 \mathrm{mg} / \mathrm{l}$ in patients with CRCL of 100 and $140 \mathrm{ml} / \mathrm{min}$, while administration of the same dose to patients with compromised renal status may lead to the accumulation of vancomycin; therefore, a dose of 400 and $600 \mathrm{mg}$ is recommended if the CRCL of the patients is 20 and $60 \mathrm{ml} / \mathrm{min}$, respectively. The dosing simulations for vancomycin in patients with estimated GFR reduction have also been previously performed in a similar study in which a 1,000-mg $\mathrm{q} 12 \mathrm{~h}$ dose of vancomycin was simulated in patients with a mild, moderate, or severe reduction in GFR (Kovacevic et al., 2020). A 


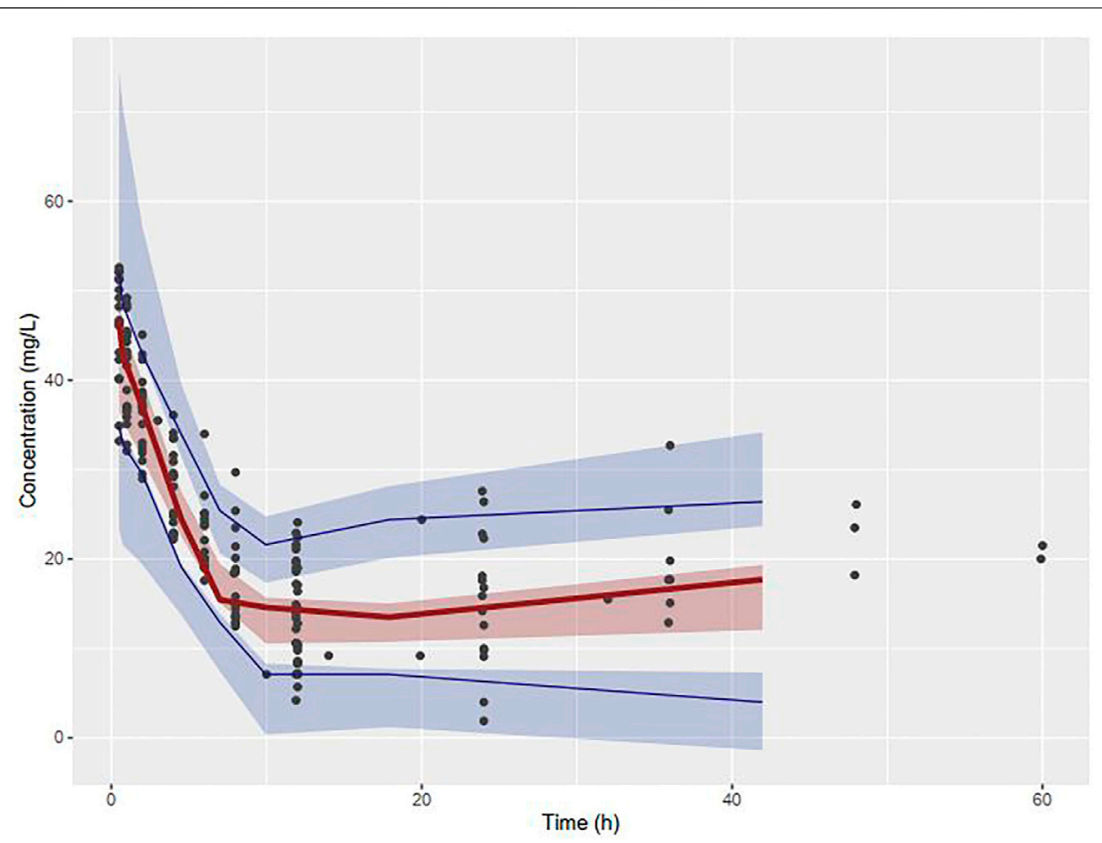

FIGURE 4 | Visual Predictive Checks of observed concentrations of vancomycin placed over the median, 5th and 95th percentiles of simulated concentrations.
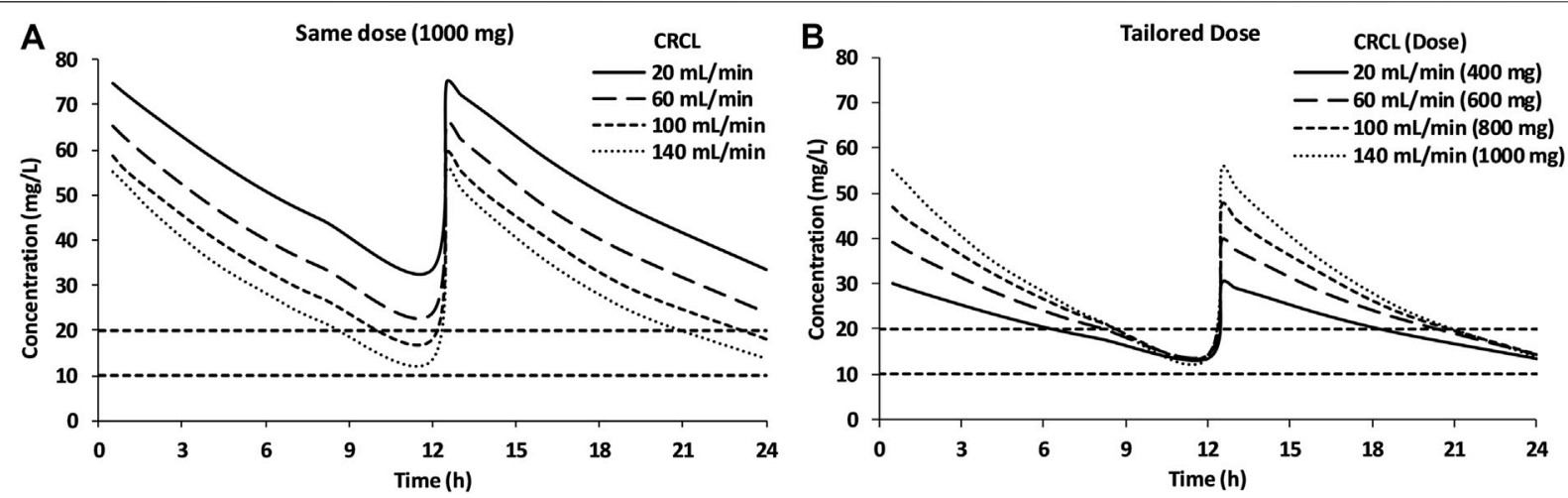

FIGURE 5 | Simulations of the 1,000-mg q12h dose for patients with different renal status (A) and simulation of tailored dose based on the renal status of the patients (B)

TABLE 3 | Trough concentrations for common and tailored doses simulated for patients with different levels of creatinine clearance.

\section{CRCL}

\section{Same dose $(\mathrm{mg})$}

1,000

1,000

1,000

1,000

\section{Simulated trough conc. (mg/L) Mean \pm S.D}

$\begin{array}{ll}20 \mathrm{ml} / \mathrm{min} & 1,000 \\ 60 \mathrm{ml} / \mathrm{min} & 1,000 \\ 100 \mathrm{ml} / \mathrm{min} & 1,000 \\ 140 \mathrm{ml} / \mathrm{min} & 1,000\end{array}$

\section{Tailored dose (mg)}

$$
\begin{array}{r}
33.3 \pm 7.54^{a} \\
23.9 \pm 5.73^{a} \\
18.0 \pm 4.56 \\
13.7 \pm 3.79
\end{array}
$$

Simulated trough conc. $(\mathrm{mg} / \mathrm{L})$ Mean \pm S.D
$13.3 \pm 3.01$
$14.4 \pm 3.44$
$14.4 \pm 3.65$
$13.7 \pm 3.79$

${ }^{a}$ Above target trough concentration range (10-20 mg/l).

dose of $1,000 \mathrm{mg}$ q12h was also simulated based on the serum cystatin $\mathrm{C}$ levels of the patients in another study (Chung et al., 2013). Furthermore, the optimal loading dose of vancomycin was estimated as $25 \mathrm{mg} / \mathrm{kg} /$ day with a maintenance dose reduced by $30 \%$ in non-dialysis patients, while in patients on dialysis, the optimum loading dose was shown to be $15 \mathrm{mg} / \mathrm{kg} /$ day with a 
maintenance dose reduced by $60 \%$ of the loading dose (Goti et al., 2018).

\section{CONCLUSION}

A population pharmacokinetic model was developed, and the pharmacokinetic parameters of vancomycin in adult Pakistani patients were comparable to those in other populations. The CRCL and body weight of patients were shown to be significant covariates for vancomycin CL in Pakistani patients. The developed model was used to simulate dosage regimens based on the CRCL of patients. The tailored doses of vancomycin in Pakistani patients were $400,600,800$, and $1,000 \mathrm{mg}$ twice daily for patients with CRCL of $20,60,100$, and $140 \mathrm{ml} / \mathrm{min}$, respectively.

\section{DATA AVAILABILITY STATEMENT}

The raw data supporting the conclusions of this article will be made available by the authors, without undue reservation.

\section{ETHICS STATEMENT}

The studies involving human participants were reviewed and approved by the Institutional Review Committee (IRC) for Biomedical Research of University of Veterinary and Animal Sciences, Lahore, Pakistan. The patients/participants provided their written informed consent to participate in this study.

\section{REFERENCES}

Aarons, L. (1991). Population Pharmacokinetics: Theory and Practice. Br. J. Clin. Pharmacol. 32, 669-670.

Abdel Hadi, O., Al Omar, S., Nazer, L. H., Mubarak, S., and Le, J. (2016). Vancomycin Pharmacokinetics and Predicted Dosage Requirements in Pediatric Cancer Patients. J. Oncol. Pharm. Pract. 22, 448-453. doi:10.1177/ 1078155215591386

Adane, E. D., Herald, M., and Koura, F. (2015). Pharmacokinetics of Vancomycin in Extremely Obese Patients with Suspected or Confirmed Staphylococcus aureus Infections. Pharmacotherapy 35, 127-139. doi:10.1002/phar.1531

Al-Kofide, H., Zaghloul, I., and Al-Naim, L. (2010). Pharmacokinetics of Vancomycin in Adult Cancer Patients. J. Oncol. Pharm. Pract. 16, 245-250. doi:10.1177/1078155209355847

Anderson, B. J., Allegaert, K., Van Den Anker, J. N., Cossey, V., and Holford, N. H. (2007). Vancomycin Pharmacokinetics in Preterm Neonates and the Prediction of Adult Clearance. Br. J. Clin. Pharmacol. 63, 75-84. doi:10.1111/j.13652125.2006.02725.x

Badran, E. F., Shamayleh, A., and Irshaid, Y. M. (2011). Pharmacokinetics of Vancomycin in Neonates Admitted to the Neonatology Unit at the Jordan University Hospital. Int. J. Clin. Pharmacol. Ther. 49, 252-257. doi:10.5414/ CP201456

Bauer, L. A., Black, D. J., and Lill, J. S. (1998). Vancomycin Dosing in Morbidly Obese Patients. Eur. J. Clin. Pharmacol. 54, 621-625. doi:10.1007/ s002280050524

Beumier, M., Roberts, J. A., Kabtouri, H., Hites, M., Cotton, F., Wolff, F., et al. (2013). A New Regimen for Continuous Infusion of Vancomycin during Continuous Renal Replacement Therapy. J. Antimicrob. Chemother. 68, 2859-2865. doi:10.1093/jac/dkt261

\section{AUTHOR CONTRIBUTIONS}

MM collected the samples, performed the popPK analysis, and drafted the manuscript. MU and HR conceived the idea and overall supervised the study. MK, RK, RB, and HS facilitated in the sample collection and compliance with ethical requirements. MAA, MOA, and AK performed the analysis of samples and proofread the manuscript. $\mathrm{HN}$ and $\mathrm{MU}$ drafted the manuscript. SA and SQ were responsible for the record keeping of plasma samples and proofreading of the manuscript.

\section{FUNDING}

The study was sponsored by the Higher Education Commission (HEC) of Pakistan via grant No. 1559/SRGP.

\section{ACKNOWLEDGMENTS}

We are grateful to the nursing staff of the Surgical Unit I of Lahore General Hospital for their help with sample collection.

\section{SUPPLEMENTARY MATERIAL}

The Supplementary Material for this article can be found online at https://www.frontiersin.org/articles/10.3389/fphar.2021.721819/ full\#supplementary-material

Blouin, R. A., Bauer, L. A., Miller, D. D., Record, K. E., and Griffen, W. O., Jr. (1982). Vancomycin Pharmacokinetics in normal and Morbidly Obese Subjects. Antimicrob. Agents Chemother. 21, 575-580. doi:10.1128/ aac. 21.4.575

Buelga, D. S., Del Mar Fernandez De Gatta, M., Herrera, E. V., Dominguez-Gil, A., and García, M. J. (2005). Population Pharmacokinetic Analysis of Vancomycin in Patients with Hematological Malignancies. Antimicrob. Agents Chemother. 49, 4934-4941. doi:10.1128/AAC.49.12.4934-4941.2005

Chen, Y., Wu, D., Dong, M., Zhu, Y., Lu, J., Li, X., et al. (2018). Population Pharmacokinetics of Vancomycin and AUC-Guided Dosing in Chinese Neonates and Young Infants. Eur. J. Clin. Pharmacol. 74, 921-930. doi:10.1007/s00228-018-2454-0

Chu, Y., Luo, Y., Ji, S., Jiang, M., and Zhou, B. (2020). Population Pharmacokinetics of Vancomycin in Chinese Patients with Augmented Renal Clearance. J. Infect. Public Health 13, 68-74. doi:10.1016/j.jiph.2019.06.016

Chung, J. Y., Jin, S. J., Yoon, J. H., and Song, Y. G. (2013). Serum Cystatin C Is a Major Predictor of Vancomycin Clearance in a Population Pharmacokinetic Analysis of Patients with normal Serum Creatinine Concentrations. J. Korean Med. Sci. 28, 48-54. doi:10.3346/jkms.2013.28.1.48

Cockcroft, D. W., and Gault, M. H. (1976). Prediction of Creatinine Clearance from Serum Creatinine. Nephron 16, 31-41. doi:10.1159/000180580

Deng, C., Liu, T., Zhou, T., Lu, H., Cheng, D., Zhong, X., et al. (2013). Initial Dosage Regimens of Vancomycin for Chinese Adult Patients Based on Population Pharmacokinetic Analysis. Int. J. Clin. Pharmacol. Ther. 51, 407-415. doi: $10.5414 / \mathrm{CP} 201842$

Dolton, M., Xu, H., Cheong, E., Maitz, P., Kennedy, P., Gottlieb, T., et al. (2010). Vancomycin Pharmacokinetics in Patients with Severe Burn Injuries. Burns 36, 469-476. doi:10.1016/j.burns.2009.08.010

Ducharme, M. P., Slaughter, R. L., and Edwards, D. J. (1994). Vancomycin Pharmacokinetics in a Patient Population: Effect of Age, Gender, and Body 
Weight. Ther. Drug Monit. 16, 513-518. doi:10.1097/00007691-19941000000013

Escobar, L., Andresen, M., Downey, P., Gai, M. N., Regueira, T., Bórquez, T., et al. (2014). Population Pharmacokinetics and Dose Simulation of Vancomycin in Critically Ill Patients during High-Volume Haemofiltration. Int. J. Antimicrob. Agents 44, 163-167. doi:10.1016/j.ijantimicag.2014.03.009

Ette, E. I., and Williams, P. J. (2004). Population Pharmacokinetics I: Background, Concepts, and Models. Ann. Pharmacother. 38, 1702-1706. doi:10.1345/ aph.1D374

Giachetto, G. A., Telechea, H. M., Speranza, N., Oyarzun, M., Nanni, L., and Menchaca, A. (2011). Vancomycin Pharmacokinetic-Pharmacodynamic Parameters to Optimize Dosage Administration in Critically Ill Children. Pediatr. Crit. Care Med. 12, e250-4. doi:10.1097/PCC.0b013e3181fe4047

Goti, V., Chaturvedula, A., Fossler, M. J., Mok, S., and Jacob, J. T. (2018). Hospitalized Patients with and without Hemodialysis Have Markedly Different Vancomycin Pharmacokinetics: A Population Pharmacokinetic Model-Based Analysis. Ther. Drug Monit. 40, 212-221. doi:10.1097/ FTD.0000000000000490

Grace, E. (2012). Altered Vancomycin Pharmacokinetics in Obese and Morbidly Obese Patients: what We Have Learned over the Past 30 Years. J. Antimicrob. Chemother. 67, 1305-1310. doi:10.1093/jac/dks066

Grimsley, C., and Thomson, A. H. (1999). Pharmacokinetics and Dose Requirements of Vancomycin in Neonates. Arch. Dis. Child. Fetal Neonatal. Ed. 81, F221-F227. doi:10.1136/fn.81.3.f221

Guilhaumou, R., Marsot, A., Dupouey, J., Galambrun, C., Boulamery, A., Coze, C., et al. (2016). Pediatric Patients with Solid or Hematological Tumor Disease: Vancomycin Population Pharmacokinetics and Dosage Optimization. Ther. Drug Monit. 38, 559-566. doi:10.1097/FTD.0000000000000318

Heffernan, A. J., Germano, A., Sime, F. B., Roberts, J. A., and Kimura, E. (2019). Vancomycin Population Pharmacokinetics for Adult Patients with Sepsis or Septic Shock: Are Current Dosing Regimens Sufficient?. Eur. J. Clin. Pharmacol. 75, 1219-1226. doi:10.1007/s00228-019-02694-1

Ingram, P. R., Lye, D. C., Tambyah, P. A., Goh, W. P., Tam, V. H., and Fisher, D. A. (2008). Risk Factors for Nephrotoxicity Associated with Continuous Vancomycin Infusion in Outpatient Parenteral Antibiotic Therapy. J. Antimicrob. Chemother. 62, 168-171. doi:10.1093/jac/dkn080

Keizer, R. J., Van Benten, M., Beijnen, J. H., Schellens, J. H., and Huitema, A. D. (2011). Piraña and PCluster: a Modeling Environment and Cluster Infrastructure for NONMEM. Comput. Methods Programs Biomed. 101, 72-79. doi:10.1016/j.cmpb.2010.04.018

Kim, D. J., Lee, D. H., Ahn, S., Jung, J., Kiem, S., Kim, S. W., et al. (2019). A New Population Pharmacokinetic Model for Vancomycin in Patients with Variable Renal Function: Therapeutic Drug Monitoring Based on Extended Covariate Model Using CKD-EPI Estimation. J. Clin. Pharm. Ther. 44, 750-759. doi:10.1111/jcpt.12995

Kimura, T., Sunakawa, K., Matsuura, N., Kubo, H., Shimada, S., and Yago, K. (2004). Population Pharmacokinetics of Arbekacin, Vancomycin, and Panipenem in Neonates. Antimicrob. Agents Chemother. 48, 1159-1167. doi:10.1128/aac.48.4.1159-1167.2004

Kovacevic, T., Miljkovic, B., Kovacevic, P., Dragic, S., Momcicevic, D., Avram, S., et al. (2020). Population Pharmacokinetic Model of Vancomycin Based on Therapeutic Drug Monitoring Data in Critically Ill Septic Patients. J. Crit. Care 55, 116-121. doi:10.1016/j.jcrc.2019.10.012

Leroux, P., Howard, M. A., 3rd, and Winn, H. R. (1990). Vancomycin Pharmacokinetics in Hydrocephalic Shunt Prophylaxis and Relationship to Ventricular Volume. Surg. Neurol. 34, 366-372. doi:10.1016/0090-3019(90) 90238-k

Li, Z. L., Liu, Y. X., Jiao, Z., Qiu, G., Huang, J. Q., Xiao, Y. B., et al. (2018). Population Pharmacokinetics of Vancomycin in Chinese ICU Neonates: Initial Dosage Recommendations. Front. Pharmacol. 9, 603. doi:10.3389/ fphar.2018.00603

Lin, W. W., Wu, W., Jiao, Z., Lin, R. F., Jiang, C. Z., Huang, P. F., et al. (2016). Population Pharmacokinetics of Vancomycin in Adult Chinese Patients with post-craniotomy Meningitis and its Application in Individualised Dosage Regimens. Eur. J. Clin. Pharmacol. 72, 29-37. doi:10.1007/s00228-015-1952-6

Lindbom, L., Pihlgren, P., Jonsson, E. N., and Jonsson, N. (2005). PsN-Toolkit--a Collection of Computer Intensive Statistical Methods for Non-linear Mixed
Effect Modeling Using NONMEM. Comput. Methods Programs Biomed. 79, 241-257. doi:10.1016/j.cmpb.2005.04.005

Llopis-Salvia, P., and Jiménez-Torres, N. V. (2006). Population Pharmacokinetic Parameters of Vancomycin in Critically Ill Patients. J. Clin. Pharm. Ther. 31, 447-454. doi:10.1111/j.1365-2710.2006.00762.x

Lo, Y. L., Van Hasselt, J. G., Heng, S. C., Lim, C. T., Lee, T. C., and Charles, B. G. (2010). Population Pharmacokinetics of Vancomycin in Premature Malaysian Neonates: Identification of Predictors for Dosing Determination. Antimicrob. Agents Chemother. 54, 2626-2632. doi:10.1128/AAC.01370-09

Machado, J. K., Feferbaum, R., Kobayashi, C. E., Sanches, C., and Santos, S. R. (2007). Vancomycin Pharmacokinetics in Preterm Infants. Clinics (Sao Paulo) 62, 405-410. doi:10.1590/s1807-59322007000400006

Mahmoud, M. A., Ebid, A. H., Shouman, S. A., and Ebid, E. N. (2014). Pharmacokinetics of Vancomycin in Oncology Egyptian Paediatrics: a Dosage Adjustment Trial. Indian J. Pharm. Sci. 76, 82-86.

Martin, J. H., Norris, R., Barras, M., Roberts, J., Morris, R., Doogue, M., et al. (2010). Therapeutic Monitoring of Vancomycin in Adult Patients: a Consensus Review of the American Society of Health-System Pharmacists, the Infectious Diseases Society of America, and the Society of Infectious Diseases Pharmacists. Clin. Biochem. Rev. 31, 21-24.

Matzke, G. R., Mcgory, R. W., Halstenson, C. E., and Keane, W. F. (1984). Pharmacokinetics of Vancomycin in Patients with Various Degrees of Renal Function. Antimicrob. Agents Chemother. 25, 433-437. doi:10.1128/ aac.25.4.433

Moffett, B. S., Ivaturi, V., Morris, J., Akcan Arikan, A., and Dutta, A. (2019a). Population Pharmacokinetic Assessment of Vancomycin Dosing in the Large Pediatric Patient. Antimicrob. Agents Chemother. 63, e02359-18. doi:10.1128/ AAC.02359-18

Moffett, B. S., Morris, J., Munoz, F., and Arikan, A. A. (2019b). Population Pharmacokinetic Analysis of Vancomycin in Pediatric Continuous Renal Replacement Therapy. Eur. J. Clin. Pharmacol. 75, 1089-1097. doi:10.1007/ s00228-019-02664-7

Moffett, B. S., Resendiz, K., Morris, J., Akcan-Arikan, A., and Checchia, P. A. (2019c). Population Pharmacokinetics of Vancomycin in the Pediatric Cardiac Surgical Population. J. Pediatr. Pharmacol. Ther. 24, 107-116. doi:10.5863/ 1551-6776-24.2.107

Montañés Pauls, B., Almiñana, M. A., and Casabó Alós, V. G. (2011). Vancomycin Pharmacokinetics during Continuous Ambulatory Peritoneal Dialysis in Patients with Peritonitis. Eur. J. Pharm. Sci. 43, 212-216. doi:10.1016/ j.ejps.2011.04.006

Omote, S., Yano, Y., Hashida, T., Masuda, S., Yano, I., Katsura, T., et al. (2009). A Retrospective Analysis of Vancomycin Pharmacokinetics in Japanese Cancer and Non-cancer Patients Based on Routine Trough Monitoring Data. Biol. Pharm. Bull. 32, 99-104. doi:10.1248/bpb.32.99

Owen, J. S., and Fiedler-Kelly, J. (2014). Introduction to Population Pharmacokinetic/Pharmacodynamic Analysis with Nonlinear Mixed Effects Models. 1st ed. Hoboken, NJ: John Wiley \& Sons, Inc.

Pakistan Bureau of Statistics (2017). Provisional Summary Result of 6th Population and Housing Census-2017. [Online]. Available: http://www.pbs.gov.pk/ content/provisional-summary-results-6th-population-and-housing-census2017-0 (Accessed March 31, 2020).

Petejova, N., Martinek, A., Zahalkova, J., Duricova, J., Brozmannova, H., Urbanek, K., et al. (2014). Vancomycin Pharmacokinetics during High-Volume Continuous Venovenous Hemofiltration in Critically Ill Septic Patients. Biomed. Pap. Med. Fac. Univ. Palacky Olomouc Czech Repub 158, 65-72. doi:10.5507/bp.2012.092

Purwonugroho, T. A., Chulavatnatol, S., Preechagoon, Y., Chindavijak, B., Malathum, K., and Bunuparadah, P. (2012). Population Pharmacokinetics of Vancomycin in Thai Patients. ScientificWorldJournal 2012, 762649. doi:10.1100/2012/762649

Revilla, N., Martín-Suárez, A., Pérez, M. P., González, F. M., and Fernández de Gatta, M. M. (2010). Vancomycin Dosing Assessment in Intensive Care Unit Patients Based on a Population Pharmacokinetic/pharmacodynamic Simulation. Br. J. Clin. Pharmacol. 70, 201-212. doi:10.1111/j.13652125.2010.03679.x

Rodvold, K. A., Blum, R. A., Fischer, J. H., Zokufa, H. Z., Rotschafer, J. C., Crossley, K. B., et al. (1988). Vancomycin Pharmacokinetics in Patients with Various 
Degrees of Renal Function. Antimicrob. Agents Chemother. 32, 848-852. doi:10.1128/aac.32.6.848

Rybak, M. J., Le, J., Lodise, T. P., Levine, D. P., Bradley, J. S., Liu, C., et al. (2020). Therapeutic Monitoring of Vancomycin for Serious Methicillin-Resistant Staphylococcus aureus Infections: A Revised Consensus Guideline and Review by the American Society of Health-System Pharmacists, the Infectious Diseases Society of America, the Pediatric Infectious Diseases Society, and the Society of Infectious Diseases Pharmacists. Am. J. Health Syst. Pharm. 77, 835-864. doi:10.1093/ajhp/zxaa036

Seay, R. E., Brundage, R. C., Jensen, P. D., Schilling, C. G., and Edgren, B. E. (1994). Population Pharmacokinetics of Vancomycin in Neonates. Clin. Pharmacol. Ther. 56, 169-175. doi:10.1038/clpt.1994.120

Staatz, C. E., Byrne, C., and Thomson, A. H. (2006). Population Pharmacokinetic Modelling of Gentamicin and Vancomycin in Patients with Unstable Renal Function Following Cardiothoracic Surgery. Br. J. Clin. Pharmacol. 61, 164-176. doi:10.1111/j.1365-2125.2005.02547.x

Stockmann, C., Hersh, A. L., Roberts, J. K., Bhongsatiern, J., Korgenski, E. K., Spigarelli, M. G., et al. (2015). Predictive Performance of a Vancomycin Population Pharmacokinetic Model in Neonates. Infect. Dis. Ther. 4, 187-198. doi:10.1007/s40121-015-0067-9

Stockmann, C., Sherwin, C. M., Zobell, J. T., Lubsch, L., Young, D. C., Olson, J., et al. (2013a). Population Pharmacokinetics of Intermittent Vancomycin in Children with Cystic Fibrosis. Pharmacotherapy 33, 1288-1296. doi:10.1002/phar.1320

Stockmann, C., Sherwin, C. M., Zobell, J. T., Lubsch, L., Young, D. C., Olson, J., et al. (2013b). Population Pharmacokinetics of Intermittent Vancomycin in Children with Cystic Fibrosis. Pharmacotherapy 33, 1288-1296. doi:10.1002/phar.1320

Taccone, F. S., Hites, M., Beumier, M., Scolletta, S., and Jacobs, F. (2011). Appropriate Antibiotic Dosage Levels in the Treatment of Severe Sepsis and Septic Shock. Curr. Infect. Dis. Rep. 13, 406-415. doi:10.1007/s11908-011-0203-y

Tanaka, A., Aiba, T., Otsuka, T., Suemaru, K., Nishimiya, T., Inoue, T., et al. (2010). Population Pharmacokinetic Analysis of Vancomycin Using Serum Cystatin C as a Marker of Renal Function. Antimicrob. Agents Chemother. 54, 778-782. doi:10.1128/AAC.00661-09

Thomson, A. H., Staatz, C. E., Tobin, C. M., Gall, M., and Lovering, A. M. (2009). Development and Evaluation of Vancomycin Dosage Guidelines Designed to Achieve New Target Concentrations. J. Antimicrob. Chemother. 63, 1050-1057. doi:10.1093/jac/dkp085

Udy, A. A., Covajes, C., Taccone, F. S., Jacobs, F., Vincent, J. L., Lipman, J., et al. (2013). Can Population Pharmacokinetic Modelling Guide Vancomycin Dosing during Continuous Renal Replacement Therapy in Critically Ill Patients? Int. J. Antimicrob. Agents 41, 564-568. doi:10.1016/j.jiantimicag.2013.01.018

Usman, M., Fobker, M., and Hempel, G. (2018). Investigation of the Age Dependency of Vancomycin Clearance by Population Pharmacokinetic Modeling. Int. J. Clin. Pharmacol. Ther. 56, 56-63. doi:10.5414/CP203033

Usman, M., and Hempel, G. (2016). Development and Validation of an HPLC Method for the Determination of Vancomycin in Human Plasma and its
Comparison with an Immunoassay (PETINIA). Springerplus 5, 124. doi:10.1186/s40064-016-1778-4

Varghese, J. M., Roberts, J. A., and Lipman, J. (2011). Antimicrobial Pharmacokinetic and Pharmacodynamic Issues in the Critically Ill with Severe Sepsis and Septic Shock. Crit. Care Clin. 27, 19-34. doi:10.1016/ j.ccc.2010.09.006

Verhave, J. C., Fesler, P., Ribstein, J., Du Cailar, G., and Mimran, A. (2005). Estimation of Renal Function in Subjects with normal Serum Creatinine Levels: Influence of Age and Body Mass index. Am. J. Kidney Dis. 46, 233-241. doi:10.1053/j.ajkd.2005.05.011

World Medical Association (2001). Decleration of Helsinki. [Online]. Available: https://www.who.int/bulletin/archives/79(4)373.pdf (Accessed March 31, 2020).

Yamamoto, M., Kuzuya, T., Baba, H., Yamada, K., and Nabeshima, T. (2009). Population Pharmacokinetic Analysis of Vancomycin in Patients with GramPositive Infections and the Influence of Infectious Disease Type. J. Clin. Pharm. Ther. 34, 473-483. doi:10.1111/j.1365-2710.2008.01016.x

Yang, R. H., Rong, X. Z., Zhang, T., and Hua, R. (2007). Changes in Pharmacokinetic Parameters of Vancomycin in the Subeschar Tissue Fluid in Patients with Severe burns. Zhonghua Shao Shang Za Zhi 23, 94-96.

Zhao, W., Zhang, D., Fakhoury, M., Fahd, M., Duquesne, F., Storme, T., et al. (2014). Population Pharmacokinetics and Dosing Optimization of Vancomycin in Children with Malignant Hematological Disease. Antimicrob. Agents Chemother. 58, 3191-3199. doi:10.1128/AAC.02564-13

Zhou, Y., Gao, F., Chen, C., Ma, L., Yang, T., Liu, X., et al. (2019). Development of a Population Pharmacokinetic Model of Vancomycin and its Application in Chinese Geriatric Patients with Pulmonary Infections. Eur. J. Drug Metab. Pharmacokinet. 44, 361-370. doi:10.1007/s13318-018-0534-2

Conflict of Interest: The authors declare that the research was conducted in the absence of any commercial or financial relationships that could be construed as a potential conflict of interest.

Publisher's Note: All claims expressed in this article are solely those of the authors and do not necessarily represent those of their affiliated organizations, or those of the publisher, the editors and the reviewers. Any product that may be evaluated in this article, or claim that may be made by its manufacturer, is not guaranteed or endorsed by the publisher.

Copyright $\odot 2021$ Munir, Rasheed, Khokhar, Khan, Saeed, Abbas, Ali, Bilal, Nawaz, Khan, Qamar, Anjum and Usman. This is an open-access article distributed under the terms of the Creative Commons Attribution License (CC BY). The use, distribution or reproduction in other forums is permitted, provided the original author(s) and the copyright owner(s) are credited and that the original publication in this journal is cited, in accordance with accepted academic practice. No use, distribution or reproduction is permitted which does not comply with these terms. 